\title{
S Research Square

\section{Phase II study of daily S-1 combined with weekly irinotecan in previously treated patients with advanced or recurrent squamous cell lung cancer: North Japan Lung Cancer Group 1101 (NJLCG1101)}

Yosuke Kawashima ( $D$ yousukekawashima3@yahoo.co.jp)

Sendai Kosei Hospital: Sendai Kosei Byoin

Osamu Ishimoto

Sendai Kosei Hospital: Sendai Kosei Byoin

Eisaku Miyauchi

Tohoku University School of Medicine: Tohoku Daigaku Daigakuin Igakukei Kenkyuka Igakubu

Tomohiro Sakakibara

Tohoku Rosai Hospital

Toshiyuki Harada

JCHO Hokkaido Hospital

Kazuhiro Usui

NTT Medical Center Tokyo

Akira Inoue

Tohoku University School of Medicine: Tohoku Daigaku Daigakuin Igakukei Kenkyuka Igakubu

Shunichi Sugawara

Sendai Kosei Hospital: Sendai Kosei Byoin

Research Article

Keywords: S-1, irinotecan, squamous cell lung cancer, Phase II trial

Posted Date: July 7th, 2021

DOl: https://doi.org/10.21203/rs.3.rs-673355/v1

License: (c) (i) This work is licensed under a Creative Commons Attribution 4.0 International License.

Read Full License 


\section{Abstract \\ Purpose}

This phase II study was designed to evaluate the efficacy and safety of S-1 combined with weekly irinotecan as second- or third-line treatment for patients with advanced or recurrent squamous cell lung cancer.

\section{Methods}

Patients received oral S-1 on days 1-14 at $80 \mathrm{mg} /$ day for patients with a body surface area of $<1.25 \mathrm{~m}^{2}$, $100 \mathrm{mg} /$ day for patients with a body surface area of $1.25-1.5 \mathrm{~m}^{2}$, and $120 \mathrm{mg} /$ day for patients with a body surface area of $>1.5 \mathrm{~m}^{2}$ and irinotecan $\left(70 \mathrm{mg} / \mathrm{m}^{2}\right)$ on days 1 and 8 every 3 weeks. The primary endpoint was overall response rate, and the secondary endpoints were progression-free survival, overall survival, and the frequency and the degree of adverse effects. The trial was registered in the University Hospital Medical Information Network Clinical Trials Registry, number UMIN000006065.

\section{Results}

Between September 2011 and December 2014, 30 patients were enrolled in this study. The overall response rate was $6.7 \%(95 \% \mathrm{Cl} 0.8-22.1)$ and the disease control rate was $73.3 \%$. The median progression-free survival was 3.0 months $(95 \% \mathrm{Cl} 2.5-3.4)$ and median overall survival was 10.5 months (95\% Cl 5.6-13.7). Grade 3 or 4 treatment-related toxicities were reported in $\geq 10 \%$ of patients including leukopenia (21\%), neutropenia (21\%), anemia (17\%), anorexia (10\%), and hypokalemia (10\%).

\section{Conclusion}

Adding weekly irinotecan to S-1 did not show the expected effect though toxicities were manageable.

\section{Introduction}

Lung cancer is one of the leading causes of cancer-related death in Japan and worldwide (Sung et al. 2021). Non-small cell lung cancer (NSCLC) accounts for $85 \%$ of all lung cancer and squamous cell carcinoma is the second most common subtype after adenocarcinoma. Squamous cell carcinoma accounts for $40 \%$ of male lung cancer and $15 \%$ of female lung cancer.

For NSCLC patients previously treated with platinum-doublet chemotherapy, single-agent chemotherapy such as pemetrexed and docetaxel are recommended. However, in terms of efficacy, pemetrexed is not recommended for patients with squamous cell carcinoma (Scagliotti et al. 2009). Furthermore, the 
efficacy of docetaxel is not satisfactory and optimization of treatment strategies for patients with squamous cell carcinoma is needed (Shepherd et al. 2000).

S-1 is a novel oral fluoropyrimidine agent that consists of tegafur, 5-chloro-2, 4-dihydroxypyridine (CDHP), and potassium oxonate in a molar ratio of 1:0.4:1. Tegafur is a prodrug of 5-fluorouracil (5-FU), whereas CDHP is an inhibitor of dihydropyrimidine dehydrogenase, the enzyme responsible for degradation of 5FU. Compared with tegafur alone, tegafur plus CDHP increases 5-FU concentration in serum and tumor tissue. Potassium oxonate is expected to palliate the gastrointestinal toxicity of tegafur. S-1 is commonly used as treatment for gastrointestinal cancer, head and neck cancer, NSCLC, breast cancer, pancreatic cancer, and biliary tract cancer. In a phase II study of S-1 monotherapy in advanced NSCLC patients without prior chemotherapy, the overall response rate (ORR) was $22.0 \%$ (95\% confidence interval [CI] 12.3-34.7\%) and there were no irreversible, severe, or unexpected toxicity (Kawahara et al. 2001). Another phase II study on S-1 monotherapy as second-line treatment for NSCLC showed an ORR of $12.5 \%(95 \% \mathrm{Cl}$ 3.1-21.9\%) and 8.2 months of median overall survival (OS) with acceptable toxicity (Totani et al. 2009). Furthermore, the phase II trial demonstrated that the combination of cisplatin plus S- 1 in advanced NSCLC patients without prior chemotherapy showed an ORR of $47 \%$ (95\% Cl 34-61\%) and 11 months OS with acceptable toxicity (Ichinose et al. 2004). A randomized phase III trial of S-1 combined with carboplatin compared with carboplatin plus paclitaxel showed noninferiority of S-1 and carboplatin in terms of OS (15.2 months vs 13.3 months, respectively; hazard ratio [HR] 0.928, 99.2\% $\mathrm{Cl} 0.671-1.283$ ) (Okamoto et al. 2010). These findings indicate that S-1 has good antitumor activity against NSCLC irrespective if given as monotherapy or combination therapy. It is also one of the good candidates for a non-platinum chemotherapy agent in patients with this disease.

Irinotecan (CPT-11) is an inhibitor of DNA topoisomerase I, which is used in the treatment of NSCLC and has different mechanism of antitumor activity from of S-1. Two randomized phase III trials of CPT-11 combined with cisplatin for advanced NSCLC showed comparable survival to cisplatin plus vindesine and concluded that a regimen containing CPT- 11 is one of the most active and well tolerated for the treatment of advanced NSCLC (Negoro et al. 2003; Niho et al. 1999). A randomized phase II trial of CPT-11 combined with docetaxel compared with cisplatin plus doxetaxel for advanced NSCLC showed no significant differences between groups in terms of OS. It was concluded that CPT-11 combined with docetaxel may be a reasonable treatment option for NSCLC patients who cannot tolerate cisplatin (Yamamoto et al. 2004).

It was suggested that thymidylate synthase (TS) expression levels in squamous cell carcinoma is higher than in adenocarcinoma, and high TS expression levels contributes to attenuation of antitumor effect. As a matter of fact, pemetrexed, which targets TS and exerts antitumor effects, should not be recommended for the treatment of squamous cell carcinoma because of the attenuation of antitumor effects (Scagliotti et al. 2009). S-1 also targets TS, however, it was suggested that there is no difference in efficacy between histological types when S-1 is combined with platinum (Yamamoto et al. 2010; Okamoto et al. 2010). For this reason, it was suggested that 5-FU has another mechanism involving RNA dysfunction by orotate phosphoribosyl transferase (OPRT) to fluorouridine monophosphate (FUMP), and high OPRT level 
contributes to enhancement of antitumor effect for metastatic colorectal cancer (Ichikawa et al. 2003). According to the investigation of OPRT levels in lung cancer, the OPRT level in squamous cell carcinoma was significantly higher than that in adenocarcinoma (Ishihama et al. 2009). Therefore, S-1 is considered to inhibit the RNA dysfunction pathway especially in squamous cell carcinoma. Furthermore, it was suggested that TS expression and topoisomerase I expression have positive correlation (Ichikawa et al. 1999), and TS expression is reduced by CPT-11 in a human colorectal cancer cell line in a preclinical model (Guichard et al. 1998). Based on these data, several studies on the combination of S-1 plus CPT-11 showed high efficacy and safety against advanced gastric and colorectal cancers (Narahara et al. 2011; Goto et al. 2006). These findings led us to investigate the possibility of using CPT- 11 combined with S-1

in patients with advanced NSCLC, especially squamous cell carcinoma. We previously reported the results of a Phase I study of daily S-1 combined with weekly CPT-11 in patients with advanced NSCLC. In this report, we found that the recommended dose (RD) of CPT-11 was $70 \mathrm{mg} / \mathrm{m}^{2}$ (Ishimoto et al. 2009). In this study, we report the results of a Phase II study of daily $S-1\left(80 \mathrm{mg} / \mathrm{m}^{2}\right.$, days $\left.1-14\right)$ combined with weekly CPT-11 (70 mg/m $\mathrm{m}^{2}$ days 1 and 8$)$ in previously treated patients with advanced squamous cell carcinoma of lung. This study was registered at UMIN-CTR under the study ID UMIN000006065.

\section{Patients And Methods Patient eligibility}

The main eligibility criteria were: (1) histologically or cytologically confirmed squamous cell lung cancer patients with a predominant squamous component or adenosquamous carcinoma with a predominant squamous component; (2) unresectable stage III disease without indication for curative irradiation, stage IV disease, or postoperative recurrence; (3) age more than 20 years old; (4) measurable lesions according to the Response Evaluation Criteria in Solid Tumors (RECIST) version 1.1 (Eisenhauer et al. 2009); (5) previously treated with less than two regimens (including at least one platinum regimen, EGFR-TKIs are counted as one regimen, post operative chemotherapy is excluded); (6) radiologically confirmed progressive disease (PD) after previous treatments (four weeks or more after previous treatment, four weeks or more after chemoradiation for locally advanced squamous cell lung cancer, two weeks or more after palliative local radiation except at the primary lesion); (7) Eastern Cooperative Oncology Group (ECOG) performance status (PS) of 0 or $1 ;$ (8) adequate organ function defined as absolute neutrophil count of $1500 \geqq \mathrm{~mm}^{3}$, platelet count $\geqq 100,000 \mathrm{~mm}^{3}$, hemoglobin $\geqq 9.0 \mathrm{~g} / \mathrm{dl}$, aspartate aminotransferase $\leqq 2.5 \times$ the institutional upper limit of the normal value, alanine aminotransferase $\leqq 2.5 \times$ the institutional upper limit of the normal value, total serum bilirubin $\leqq 1.5 \times$ the institutional upper limit of the normal value, creatinine $\leqq 1.2 \mathrm{mg} / \mathrm{dl}$, creatinine clearance $\geq 60 \mathrm{~mL} / \mathrm{min}, \mathrm{PaO}_{2} \geqq 60$ Torr or $\mathrm{SpO}_{2} \geqq 94 \%$; and (9) written informed consent.

The main exclusion criteria were: (1) evidence of interstitial pneumonia on chest radiography; (2) past history of hypersensitivity to drugs; (3) active double cancer; (4) pleural, peritoneal, and pericardial effusion requiring drainage; (5) serious complications (symptomatic cardiovascular disease, uncontrolled 
hypertension and diabetes, active infections); (6) symptomatic brain metastases; (7) previously treated with S-1, UFT, and CPT-11; (8) water diarrhea; (9) intestinal paralysis or intestinal obstruction; (10) treated with flucytosine; (11) treated with atazanavir sulfate; and (12) women who were pregnant, intending to become pregnant, or breast-feeding. The study protocol was approved by the institutional review board of each participating hospital and written informed consent was obtained from all patients prior to enrollment.

\section{Treatment schedule}

Every 21-day cycle, intravenous CPT-11 $\left(70 \mathrm{mg} / \mathrm{m}^{2}\right)$ was administered on days 1 and 8 , and oral S-1 was administered twice daily after a meal from days 1 to 14 . The dose of S-1 was modified according to body surface area (BSA) as follows: $80 \mathrm{mg} /$ day for patients with a BSA less than $1.25 \mathrm{~m}^{2}, 100 \mathrm{mg} /$ day for those with a BSA from 1.25 to $1.5 \mathrm{~m}^{2}$, and $120 \mathrm{mg}$ /day for those with a BSA of more than $1.5 \mathrm{~m}^{2}$. The criteria for initiating the treatment cycle were provided, and cycle delays of up to three weeks were permitted. The criteria for giving CPT-11 on day 8 were provided and the administration of CPT-11 was skipped if the patient did not meet the criteria. The criteria for S-1 cessation and resumption were also provided. The dose reduction criteria were as follows. The dose of S-1 was reduced at first dose reduction as follows: from 120 to $100 \mathrm{mg} /$ day, from 100 to $80 \mathrm{mg} /$ day, and from 80 to $50 \mathrm{mg} /$ day. The dose of CPT11 was reduced at second dose reduction as follows: from $70 \mathrm{mg} / \mathrm{m}^{2}$ to $60 \mathrm{mg} / \mathrm{m}^{2}$. Patients who required dose reduction received the reduced dose for the rest of the study. If a patient who required second dose reduction became eligible for further dose reduction, the patient was withdrawn from the study.

\section{Assessment of Endpoints}

The primary endpoint was investigator-assessed ORR, which was defined as the proportion of confirmed complete response (CR) or a partial response (PR). Tumor response assessments were performed by using spiral computed tomography (CT) and evaluated using RECIST version 1.1 every four weeks from screening until PD was observed. After the discontinuation of treatment and the tumor response was confirmed as CR, PR, or stable disease (SD), CT was performed every eight weeks. The secondary endpoints were progression-free survival (PFS), OS, disease control rate (DCR), and toxicity. PFS was defined as the time from randomization to disease progression according to RECIST version 1.1 or death due to any cause, whichever occurred first. OS was defined as the time from randomization to death due to any cause. The DCR was defined as the proportion of occurrence of CR, PR, or SD according to RECIST version 1.1. The toxicities were assessed according to Common Terminology Criteria for Adverse Events (CTCAE) version 4.0. The investigators determined whether the toxicities were caused by the trial regimen.

\section{Statistical Analysis}

Several phase III studies on docetaxel and erlotinib with patients who were previously treated with platinum showed that the ORR was from 5.5-12.8\%. Combination therapy containing CPT-11 given to patients who was previously treated with platinum showed that the ORR was from $10-20 \%$. Based on 
these data, we assumed that an ORR of $20 \%$ in eligible patients indicates potential usefulness and an ORR of $5 \%$ is the lower limit of interest. Accordingly, the estimated accrual using the SWOG one arm binomial was 28 patients in each arm (one-sided alpha $=0.05$; beta $=0.20$ ). After allowing for dropouts, the accrual goal was determined to be 30 patients. The PFS and OS were estimated until 31 December 2019 by using the Kaplan-Meier method and were analyzed using the log-rank test. All statistical analyses were performed with EZR (Saitama Medical Centre, Jichi Medical University, Saitama, Japan).

\section{Results}

\section{Patient characteristics}

Between September 2011 and December 2014, 30 patients were enrolled. The baseline characteristics of the patients are summarized in Table 1. The median patient age was 65 years (range; 47-77), and 23 patients $(76.7 \%)$ were men. Twenty-five patients $(83.3 \%)$ were previously treated with one regimen.

\section{Treatment delivery}

At the time of the data cutoff date (March 31, 2019) for the final analyses, all patients finished receiving treatment. The median number of treatment courses was three (range; 1-18). A total of 3 patients (10.0\%) required dose reduction of CPT-11, and 15 patients (50.0\%) required a delay in the treatment cycles. The administration of CPT-11 on day 8 was skipped among 3 patients $(10.0 \%)$. A total of six patients $(20.0 \%)$ required dose reduction in S-1, while seven patients $(23.3 \%)$ required cessation of S-1 in the treatment cycles (Table 2). The median relative dose intensities were $83.3 \%$ (CPT-11) and $84.9 \%$ (S-1), respectively.

\section{Efficacy}

Tumor response was evaluated in all patients enrolled in the study and the ORR was $6.7 \%(95 \% \mathrm{Cl} 0.8-$ 22.1) while the DCR was $73.3 \%$ ( $95 \% \mathrm{Cl} 54.1-87.7)$. Tumor response in the study is summarized in Table 3. Median progression-free survival was 3.0 months $(95 \% \mathrm{Cl} 2.5-3.4)$ and median overall survival was 10.5 months (95\% Cl 5.6-13.7), respectively (Figure 1, 2).

Safety

One patient was excluded due to a lack of data and 29 patients were included in the safety analysis. Adverse events that occurred in the study are listed in Table 4. Grade 3 or 4 hematological toxicities included leukopenia (21\%), neutropenia (21\%), and anemia (17\%). Grade 3 or 4 non-hematological toxicities included anorexia (10\%), nausea (3\%), diarrhea (7\%), oral mucositis (3\%), peripheral neuropathy (3\%), febrile neutropenia (3\%), hypoalbuminemia (3\%), hyponatremia $(7 \%)$, and hypokalemia $(10 \%)$. There was no treatment-related death.

\section{Discussion}


In the present study, we evaluated the efficacy and safety of CPT-11 plus S-1 in previously treated patients with advanced or recurrent squamous cell lung cancer. We obtained an ORR of $6.7 \%$, while the median PFS and OS were 3.0 months and 10.5 months, respectively. To the best of our knowledge, there are two studies evaluating the efficacy and safety of CPT-11 plus S-1 in patients with previously treated advanced or recurrent NSCLC. Goya et al. reported that the ORR was $15.8 \%(90 \% \mathrm{Cl} 6.1-25.5)$ and the median PFS and OS were 4.5 months $(95 \% \mathrm{Cl} 3.5-5.0)$ and 15.0 months (95\% Cl 9.5-20.6), respectively (2012). Ikeumura et al. reported that the ORR was $6.5 \%(95 \% \mathrm{Cl}-2.6-15.5)$ and the median PFS and OS were 2.8 months (95\% Cl 2.3-3.4) and 12.6 months (95\% Cl 8.9-19.9), respectively (2015). In both studies, the dominant histological type was adenocarcinoma and a limited number of squamous cell lung cancer patients were included ( $15.8 \%$ and $22.6 \%$, respectively). Therefore, our study is worthy of evaluating the efficacy and safety of CPT-11 plus S-1 particularly in patients with squamous cell lung cancer.

Although we planned that the CPT-11 will be given at a dose of $70 \mathrm{mg} / \mathrm{m}^{2}$ on days 1 and 8 every 3 weeks ( $=46.7 \mathrm{mg} / \mathrm{m}^{2} /$ week) according to the result of the Phase I study of daily S-1 combined with weekly CPT11 in patients with advanced NSCLC, the dominant histological type was adenocarcinoma and only one patient (7.7\%) with squamous cell lung cancer was included in the study (Ishimoto et al. 2009). In the current study, the median relative dose intensity of CPT-11 was $83.3 \%$ and the actual dose intensity of CPT-11 was $38.9 \mathrm{mg} / \mathrm{m}^{2} /$ week. The histological type and the discrepancy in the dose intensity of CPT-11 may have contributed to a lower ORR than what we expected.

The toxicity profile of the adverse events was as expected. For instance, 6/29 (20.7\%) patients experienced grade 3-4 leukopenia or neutropenia, which was consistent with previous studies with second-line treatment. In these studies, the rate of grade 3 or 4 neutropenia in patients treated with CPT11 and S-1 combination was 9.7-17.9\%. With respect to non-hematological toxicities, grade 3 or 4 diarrhea occurred in 2/29 (6.9\%) patients, which seems to be within the acceptable range. One adverse event to note is that $5 / 29(17.2 \%)$ experienced grade $3-4$ anemia. Although no patient developed grade 4 anemia, we should keep in mind that anemia sometimes can be life threatening.

There are several limitations in this study. First, immunotherapy was introduced into clinical practice after this study and no patient was treated with immunotherapy as the initial therapy. Immune checkpoint inhibitors (ICl) monotherapy, ICl plus chemotherapy, and ICl combination therapy are standard treatment for advanced NSCLC these days. Therefore, our study does not reflect the situation of current advances in immunotherapy. Second, we had planned this study based on the hypothesis that the OPRT expression and TS expression of squamous cell carcinoma was different from that of adenocarcinoma, however, we did not evaluate the actual OPRT expression and TS expression of tumors.

In conclusion, CPT-11 plus S-1 does not surpass the results of the single agent regarding ORR. This combination could not be a candidate regimen for further phase III studies. On the other hand, the toxicity profile was as expected, and this regimen might be considered if combination regimen of non-platinum agent were preferred because platinum-based regimen is sometimes toxic and intolerable. 


\section{Declarations}

\section{Acknowledgments}

The authors thank all patients, families, and investigators participating in the NJLCG 1101 study.

\section{Funding}

This research did not receive any specific grant from funding agencies in the public, commercial, or notfor-profit sectors.

\section{Conflict of interest statement}

Dr. Miyauchi reports personal fees from TAlHO Phamaceutical Co., Ltd. outside the submitted work; Dr. Inoue reports personal fees from Daiichi Sankyo outside the submitted work; Dr. Sugawara reports personal fees from Taiho Pharmaceutical, personal fees from Chugai Pharma, personal fees from AstraZeneca, personal fees from MSD, personal fees from Bristol-Myers Squibb, personal fees from Ono Pharmaceutical, personal fees from Nippon Boehringer Ingelheim, personal fees from Pfizer, personal fees from Eli Lilly and Company, personal fees from Novartis, personal fees from Kyowa Kirin, and personal fees from Yakult Honsha outside the submitted work; All other authors declare no conflict of interest.

\section{Ethical Approval and consent to participate}

This study protocol was reviewed and approved by the institutional review boards of the participating institutions and written informed consent was obtained from all patients prior to enrollment.

\section{Consent for publication}

Not applicable.

\section{Data availability}

All data generated or analyzed during this study are included in this published article.

\section{Authors' Contributions}

Dr. Kawashima and Sugawara have full access to all the data in the study and take responsibility for the integrity of the data and the accuracy of the data analysis. All authors contributed to patient recruitment and data collection. All authors read and approved the final manuscript

\section{References}

1. Eisenhauer EA, Therasse P, Bogaerts $\mathrm{J}$ et al (2009) New response evaluation criteria in solid tumours: revised RECIST guideline (version 1.1). Eur J Cancer 45:228-247 
2. Goto A, Yamada Y, Yasui H et al (2006) Phase II study of combination therapy with S-1 and irinotecan in patients with advanced colorectal cancer. Ann Oncol 17:968-973

3. Goya H, Kuraishi H, Koyama S et al (2012) Phase I/II study of S-1 combined with biweekly irinotecan chemotherapy in previously treated advanced non-small cell lung cancer. Cancer Chemother Pharmacol 70:691-697

4. Guichard S, Hennebelle I, Bugat R, Canal P (1998) Cellular interactions of 5-fluorouracil and the camptothecin analogue CPT-11 (irinotecan) in a human colorectal carcinoma cell line. Biochem Pharmacol 55:667-676

5. Ichikawa W, Uetake H, Nihei Z et al. Topoisomerase I (Topo-I) expression correlates to thymidylate synthase (TS) expression in colorectal cancer (CRC). Proc Am Soc Clin Oncol 1999;18;246a, (abstr 946)

6. Ichikawa W, Uetake H, Shirota Y et al (2003) Both gene expression for orotate phosphoribosyltransferase and its ratio to dihydropyrimidine dehydrogenase influence outcome following fluoropyrimidine-based chemotherapy for metastatic colorectal cancer. Br J Cancer 89:1486-1492

7. Ichinose Y, Yoshimori K, Sakai H et al (2004) S-1 plus cisplatin combination chemotherapy in patients with advanced non-small cell lung cancer: a multi-institutional phase II Trial. Clin Cancer Res 10:7860-7864

8. Ikemura S, Naoki K, Yasuda $\mathrm{H}$ et al (2015) A phase II study of S-1 and irinotecan combination therapy in previously treated patients with advanced non-small cell lung cancer. Jpn J Clin Oncol 45:356353

9. Ishihama H, Chida M, Araki $\mathrm{O}$ et al (2009) Comparison of 5-fluorouracil-related gene expression levels between adenocarcinomas and squamous cell carcinomas of the lung. Jpn J Clin Oncol 39:33-36

10. Ishimoto O, Ishida T, Honda Y, Munakata M, Sugawara S (2009) Phase I study of daily S-1 combined with weekly irinotecan in patients with advanced non-small cell lung cancer. Int J Clin Oncol 14:4347

11. Kawahara M, Furuse K, Segawa Y et al (2001) Phase Il study of S-1, a novel oral fluorouracil, in advanced non-small-cell lung cancer. $\mathrm{Br} \mathrm{J}$ Cancer 85:939-943

12. Narahara $\mathrm{H}$, lishi $\mathrm{H}$, Imamura $\mathrm{H}$ et al (2011) Randomized phase III study comparing the efficacy and safety of irinotecan plus S-1 with S-1 alone as first-line treatment for advanced gastric cancer (study GC0301/TOP-002). Gastric cancer 14:72-80

13. Negoro S, Masuda N, Takada Y et al (2003) Randomised phase III trial of irinotecan combined with cisplatin for advanced non-small-cell lung cancer. Br J Cancer 88:335-341

14. Niho S, Nagao K, Nishiwaki Y et al (1999) Randomized multicenter phase III trial of irinotecan (CPT11 ) and cisplatin (CDDP) versus CDDP and vindesine (VDS) in patients with advanced non small cell lung cancer (NSCLC). Proc Am Soc Clin Oncol 18(a):492, (abstr 1897)

15. Okamoto I, Yoshioka H, Morita S et al (2010) Phase III trial comparing oral S-1 plus carboplatin with paclitaxel plus carboplatin in chemotherapy-naïve patients with advanced non-small-cell lung cancer: 
results of a west Japan oncology group study. J Clin Oncol 28:5240-5246

16. Scagliotti G, Hanna N, Fossella F et al. The differential efficacy of pemetrexed according to NSCLC histology: a review of two Phase III Studies. Oncologist 2009;14:253 - 63

17. Shepherd FA, Dancey J, Ramlau R et al (2000) Prospective randomized trial of docetaxel versus best supportive care in patients with non-small-cell lung cancer previously treated with platinum-based chemotherapy. J Clin Oncol 18:2095-2103

18. Sung H, Ferlay J, Siegel RL et al (2021) Global Cancer Statistics 2020: GLOBOCAN estimates of incidence and mortality worldwide for 36 cancers in 185 countries. CA Cancer J Clin 0:1-41

19. Totani Y, Saito Y, Hayashi M et al (2009) A phase Il study of S-1 monotherapy as second-line treatment for advanced non-small cell lung cancer. Cancer chemother Pharmacol 64:1181-1185

20. Yamamoto N, Fukuoka M, Negoro SI et al (2004) Randomized phase II study of docetaxel/cisplatin vs docetaxel/irinotecan in advanced non-small-cell lung cancer: a West Japan Thoracic Oncology Group Study (WJTOG9803). Br J Cancer 90:87-92

21. Yamamoto N, Yamanaka T, Ichinose $Y$ et al (2010) Pooled analysis of S-1 trials in non-small cell lung cancer according to histological type. Anticancer Res 30:2985-2990

\section{Tables}

Table 1. Patient characteristics

\begin{tabular}{|lr|}
\hline No. of patients & \multicolumn{1}{l|}{30} \\
\hline Age (years), median (range) & $65(47-77)$ \\
\hline Gender & $23(76.7 \%)$ \\
Male & $7(23.3 \%)$ \\
Female & \\
\hline Performance status (ECOG) & $16(53.3 \%)$ \\
0 & $14(46.7 \%)$ \\
1 & \\
\hline Number of previous regimens & $25(83.3 \%)$ \\
\hline 1 & $5(16.7 \%)$ \\
\hline 2 & \\
\hline
\end{tabular}

Abbreviations: ECOG, Eastern Cooperative Oncology Group

Table 2. Treatment delivery

The median number of treatment courses was three (range; 1-18) 


\begin{tabular}{|lll|}
\hline & S-1 & CPT-11 \\
\hline Dose reduction & $6(20.0 \%)$ & $3(10.0 \%)$ \\
\hline Course delay & $15(50.0 \%)$ & $15(50.0 \%)$ \\
\hline Cessation & $7(23.3 \%)$ & - \\
\hline Skip of CPT-11 on day 8 & - & $3(10.0 \%)$ \\
\hline The relative median dose intensity (\%) & $84.9 \%$ & $83.3 \%$ \\
\hline
\end{tabular}

Table 3. Response to Treatment

\begin{tabular}{|c|c|c|}
\hline & No. of patients & $(\%)$ \\
\hline Complete response & 0 & 0 \\
\hline Partial response & 2 & 6.7 \\
\hline Stable disease & 20 & 66.7 \\
\hline Progressive disease & 7 & 23.3 \\
\hline Not evaluable & 1 & 3.3 \\
\hline Overall response rate & & 6.7 \\
\hline Disease control rate & & 73.3 \\
\hline
\end{tabular}

Table 4. Hematological and non-hematological toxicities 


\begin{tabular}{|lllll|}
\hline Toxicity & All events & Grade 3 & Grade 4 & Grade 3/4 \\
& $\mathrm{n}(\%)$ & $\mathrm{n}(\%)$ & $\mathrm{n}(\%)$ & $\%$ \\
\hline Hematologic & & & & \\
Leucopenia & $10(34.5 \%)$ & $5(17.2 \%)$ & $1(3.4 \%)$ & $20.7 \%$ \\
\hline Neutropenia & $12(41.4 \%)$ & $1(3.4 \%)$ & $5(17.2 \%)$ & $20.7 \%$ \\
\hline Anemia & $26(89.7 \%)$ & $5(17.2 \%)$ & $0(0.0 \%)$ & $17.2 \%$ \\
\hline Thrombocytopenia & $9(31.0 \%)$ & $0(0.0 \%)$ & $0(0.0 \%)$ & $0.0 \%$ \\
\hline Non-hematologic & & & & \\
\hline Fatigue & $3(10.3 \%)$ & $0(0.0 \%)$ & $0(0.0 \%)$ & $0.0 \%$ \\
\hline Anorexia & $15(51.7 \%)$ & $3(10.3 \%)$ & $0(0.0 \%)$ & $10.3 \%$ \\
\hline Nausea & $8(27.6 \%)$ & $1(3.4 \%)$ & $0(0.0 \%)$ & $3.4 \%$ \\
\hline Diarrhea & $11(37.9 \%)$ & $2(6.9 \%)$ & $0(0.0 \%)$ & $6.9 \%$ \\
\hline Oral mucositis & $2(6.9 \%)$ & $1(3.4 \%)$ & $0(0.0 \%)$ & $3.4 \%$ \\
\hline Peripheral neuropathy & $2(6.9 \%)$ & $1(3.4 \%)$ & $0(0.0 \%)$ & $3.4 \%$ \\
\hline Febrile neutropenia & $1(3.4 \%)$ & $1(3.4 \%)$ & $0(0.0 \%)$ & $3.4 \%$ \\
\hline Hypoalbuminemia & $6(20.7 \%)$ & $1(3.4 \%)$ & $0(0.0 \%)$ & $3.4 \%$ \\
\hline Creatinine increased & $5(17.2 \%)$ & $0(0.0 \%)$ & $0(0.0 \%)$ & $0.0 \%$ \\
\hline Hyponatremia & $4(13.8 \%)$ & $2(6.9 \%)$ & $0(0.0 \%)$ & $6.9 \%$ \\
\hline Hypokalemia & $7(24.1 \%)$ & $2(6.9 \%)$ & $1(3.4 \%)$ & $10.3 \%$ \\
\hline
\end{tabular}

Data are No. (\%)

\section{Figures}




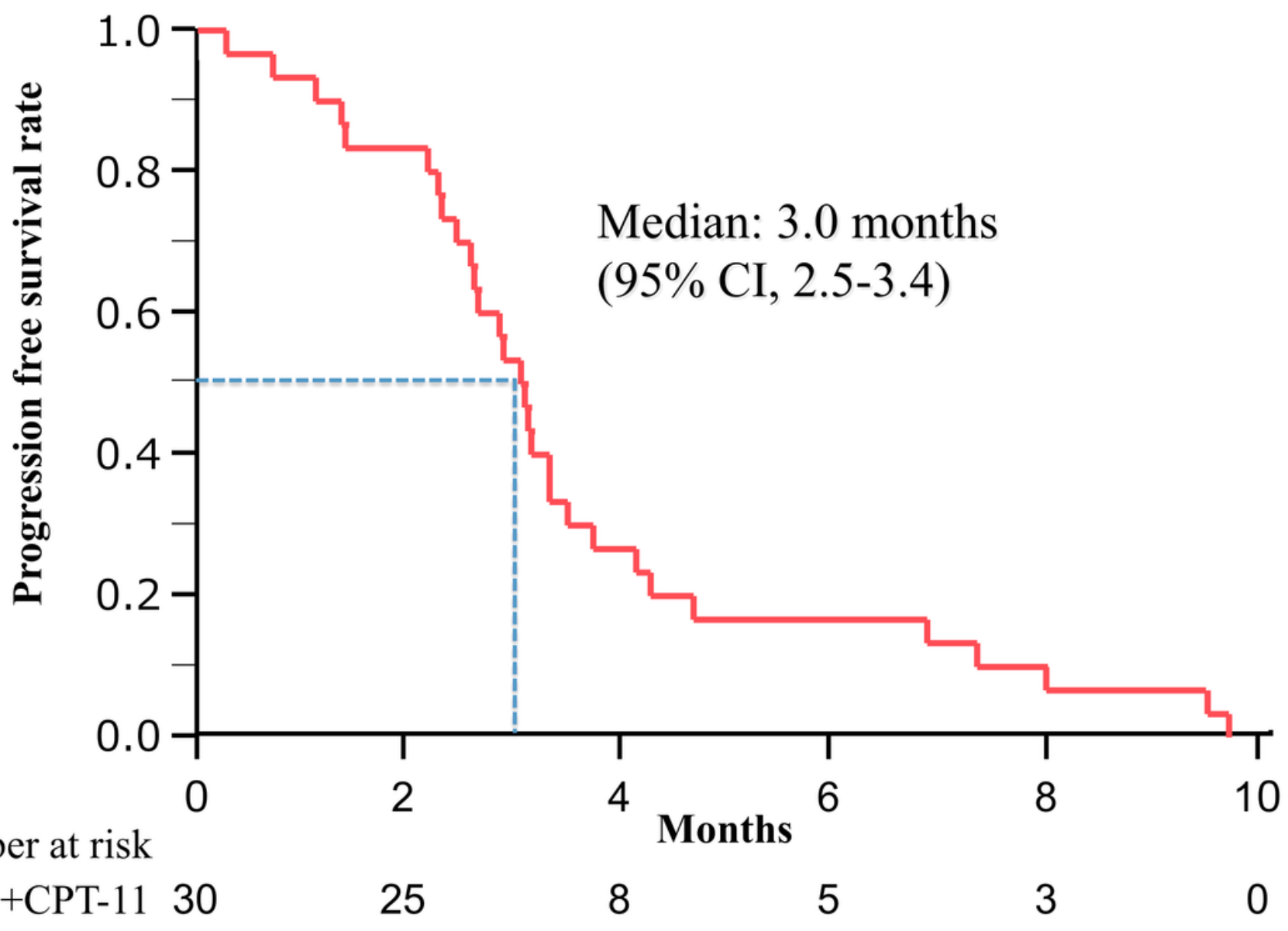

Figure 1

Kaplan-Meier analysis of progression-free survival Ticks indicate patients for whom data were censored on March 31, $2019 \mathrm{Cl}$, confidence interval 


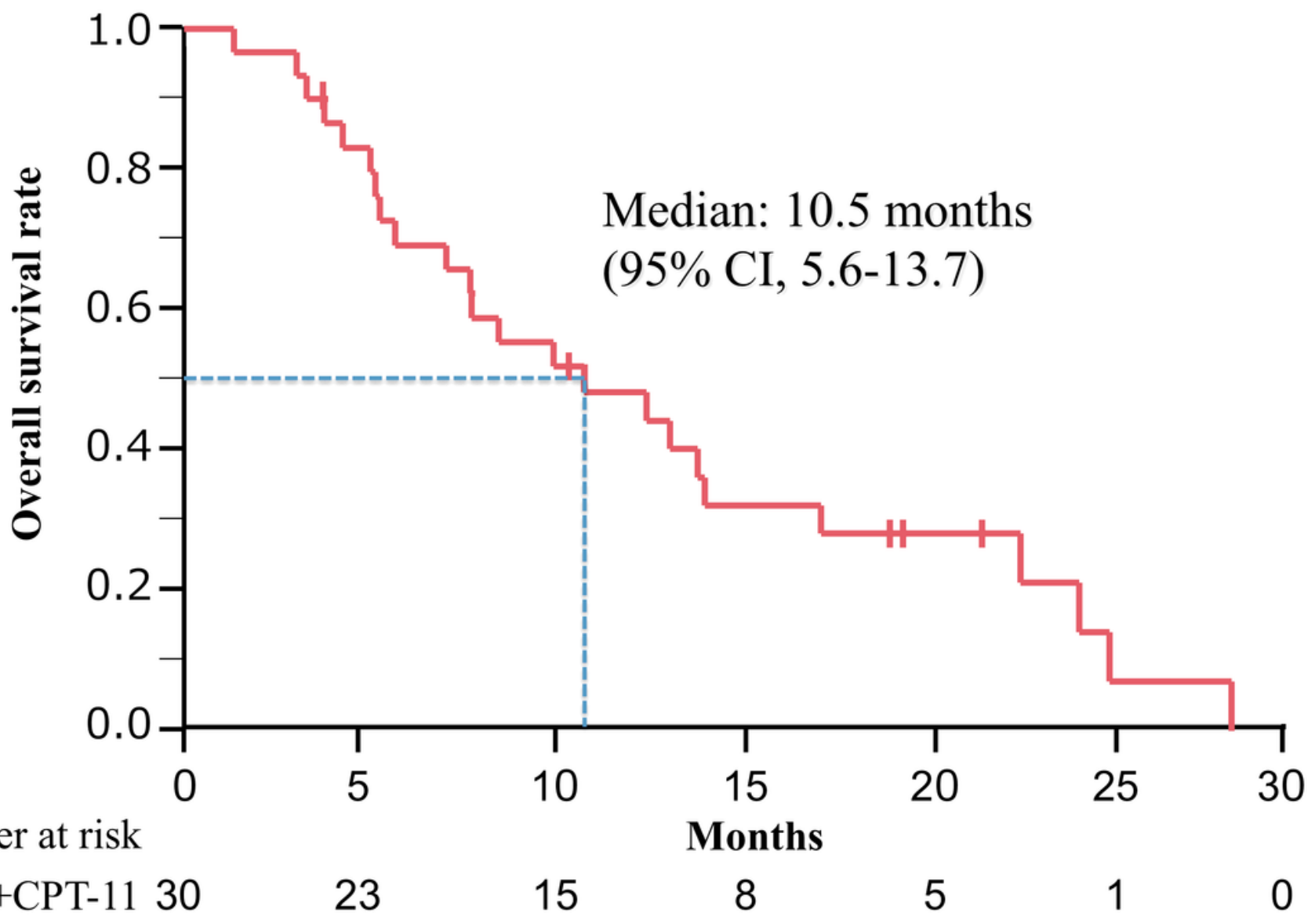

Figure 2

Kaplan-Meier analysis of overall survival Ticks indicate patients for whom data were censored on March $31,2019 \mathrm{Cl}$, confidence interval 